\title{
Quantum criticality in disordered bosonic optical lattices
}

\author{
Xiaoming Cai, ${ }^{1}$ Shu Chen,, , ${ }^{*}$ and Yupeng Wang ${ }^{1}$ \\ ${ }^{1}$ Beijing National Laboratory for Condensed Matter Physics, \\ Institute of Physics, Chinese Academy of Sciences, Beijing 100190, China
}

(Dated: March 17, 2021)

\begin{abstract}
Using the exact Bose-Fermi mapping, we study universal properties of ground-state density distributions and finite-temperature quantum critical behavior of one-dimensional hard-core bosons in trapped incommensurate optical lattices. Through the analysis of universal scaling relations in the quantum critical regime, we demonstrate that the superfluid to Bose glass transition and the general phase diagram of disordered hard-core bosons can be uniquely determined from finite-temperature density distributions of the trapped disordered system.
\end{abstract}

PACS numbers: 05.30.Rt ,05.30.Jp ,72.15.Rn ,03.75.Hh

Introduction.- In recent years ultracold atomic systems have proven to be powerful quantum simulators for investigating various challenging many-body physics. In comparison with traditional condensed matter systems, both the potential and interaction of trapped atomic systems can be experimentally implemented in a controlled way, which leads to experimental breakthroughs in the study of fundamental model systems, including the realization of the Tonks-Girardeau (TG) gas [1 3 ], and the observation of Anderson localization in disordered bosonic optical lattices [4 7]. The interplay of disorder and interactions for bosons has often been studied in the context of Bose-Hubbard model with disordered or incommensurate potentials. Theoretical studies have indicated that a superfluid to Bose-glass (BG) transition occurs for increasing disorder [8, 9]. Despite intensive studies [8-11], no exact solutions for disordered interacting bosons are known except that in the TG limit the one-dimensional (1D) disordered Bose-Hubbard models are exactly solvable via a Bose-Fermi mapping [12 14].

So far most of theoretical studies on 1D disordered bosonic gases focused on zero temperature cases, as finite-temperature phase transitions are generally absent in 1D systems. However, considering realistic experiments are always carried on at finite temperature for trapped systems, it is important to understand how to unambiguously determine the zero temperature phase diagram from the knowledge of finite temperature density profiles of trapped gases [15 17]. In the presence of a harmonic trap, Anderson plateaus are found in the average density profile of the hard-core bosons in the incommensurate optical lattice 14]. The Anderson plateau can be viewed as the signature of Anderson localized states with superfluid to BG transitions taking place at edges of the plateau. At finite temperatures, Anderson plateaus are smeared out by thermal fluctuations and thus no sharp boundaries for superfluid and BG phases could be detected. In order to understand zero temperature phase transitions, we must consider the so-called quantum critical regime where some universal scaling relations govern the finite-temperature physics. In principle, one can extract the zero-temperature phase diagram of the disordered Bose system from these universal relations. Unfortunately, it is very hard to calculate these universal functions due to lack of reliable general techniques for disordered many-body problems. Since quantum and thermal fluctuations strongly couple together in the quantum critical regime, highly precise calculation of the finitetemperature data is needed in order to extract correct zero-temperature phase diagram. In this work, by using the exact numerical method based on the Bose-Fermi mapping, we can calculate finite-temperature properties of disordered hard-core bosons exactly, and thus demonstrate that the zero-temperature phase diagram can be uniquely determined from finite-temperature density distributions of the trapped gas.

To make progress in unveiling the quantum criticality in disordered Bose systems quantitatively, we study a disordered 1D TG gas with the effect of disorder being mimicked by an incommensurate potential [5, 6], which is described by

$$
H=-t \sum_{i}\left(\hat{b}_{i}^{\dagger} \hat{b}_{i+1}+\text { H.c. }\right)+\sum_{i} V_{i} \hat{n}_{i},
$$

where $\hat{b}_{i}^{\dagger}\left(\hat{b}_{i}\right)$ is the creation (annihilation) operator of the boson fulfilling the hard-core constraints, i.e., the on-site anticommutation $\left(\left\{\hat{b}_{i}, \hat{b}_{i}^{\dagger}\right\}=1\right)$ and $\left[\hat{b}_{i}, \hat{b}_{j}^{\dagger}\right]=0$ for $i \neq j$, and $\hat{n}_{i}=\hat{b}_{i}^{\dagger} \hat{b}_{i}$. The hopping amplitude $t$ is set to be the unit of the energy $(t=1)$, and $V_{i}$ is given by

$$
V_{i}=V_{I} \cos (\alpha 2 \pi i+\delta)+V_{H}\left(i-i_{0}\right)^{2} .
$$

Here $V_{I}$ is the strength of incommensurate potentia with $\alpha$ being an irrational number and $\delta$ an arbitrary phase which is chosen to be zero for convenience, and $V_{H}$ is the strength of the additional harmonic trap with $i_{0}$ being the position of trap center. In the TG limit, the Hamiltonian (1) can be mapped to a noninteracting spinless Fermi model, which allows us to calculate even the finite-temperature properties by using the exact numerical method [18, 19]. 


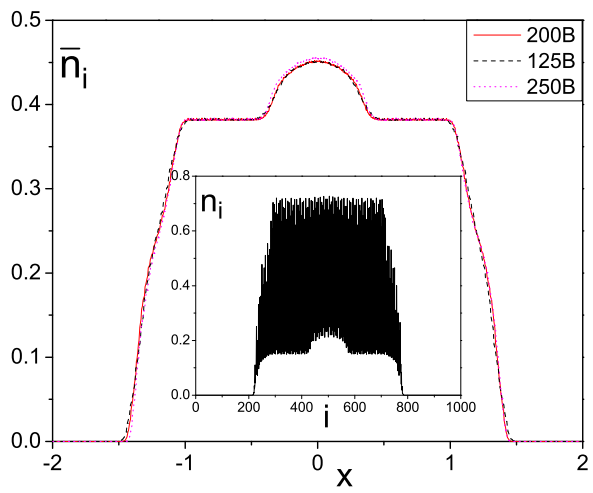

FIG. 1: (Color online) Local average density profiles relative to the scaled position for different systems with same characteristic density $(\widetilde{\rho}=1)$. The three systems are: 200 bosons, $V_{H}=2.5 \times 10^{-5} ; 125$ bosons, $V_{H}=6.4 \times 10^{-5}$; and 250 bosons, $V_{H}=1.6 \times 10^{-5}$ with 1000 sites, $V_{I}=1$ and $\alpha=(\sqrt{5}-1) / 2$. Insert: corresponding density profile for the system with 200 bosons.

Universal properties of zero-temperature density distribution.- In order to characterize universal features of lattice systems in harmonic traps, we make use of the length scale $\zeta=\left(V_{H} / t\right)^{-1 / 2}$ and the characteristic density $\widetilde{\rho}=N / \zeta$ introduced in Ref. [20]. Without the incommensurate potential the system has been studied by Rigol and Muramatsu in detail [18], and they found that there is a critical characteristic density in the system $\left(\widetilde{\rho}_{c} \sim 2.6-2.7\right)$. For low characteristic density( $\widetilde{\rho}<\widetilde{\rho}_{c}$ ), the whole system is in a uniform phase (superfluid phase) at zero temperature, and for $\widetilde{\rho}>\widetilde{\rho}_{c}$ two different phases coexist with a Mott insulating plateau in the middle of the trap surrounded by superfluid phases on two sides. In the presence of incommensurate potential [14], the density profiles basically still have the arc shape for weak $V_{I}$, but there are a lot of drastic oscillations induced by the incommensurate potential (see the insert picture in Fig[1). In order to reduce the drastic oscillations in density profiles, we define the local average density $\bar{n}_{i}=\sum_{j=-M}^{M} n_{i+j} /(2 M+1)$, where $2 M+1$ is the length to count the local average density with $M \ll L$ and $n_{i}=\left\langle\Psi_{\mathrm{HCB}}^{G}\left|\hat{n}_{i}\right| \Psi_{\mathrm{HCB}}^{G}\right\rangle$ with $\Psi_{\mathrm{HCB}}^{G}$ the ground state of the Hamiltonian (11). When $V_{I} \rightarrow 0$, the local average density profile is almost the same as the density profile. As $V_{I}$ increases, Anderson plateaus appear at shoulders of the arc, then become wider and wider but the height of the plateau does not change with $V_{I}$. For sites in the Anderson plateau the one particle density matrices $\rho_{i j}=\left\langle\Psi_{\mathrm{HCB}}^{G}\left|\hat{b}_{i}^{\dagger} \hat{b}_{j}\right| \Psi_{\mathrm{HCB}}^{G}\right\rangle$ have an exponential-law decay which is the character of the particle in the BG phase. While for sites outside the plateau, $\rho_{i j}$ exhibit a power-law decay which is the character of the particle in the superfluid phase.

Now we discuss the universal properties of Anderson plateaus. In Fig 11 we show local average density pro-

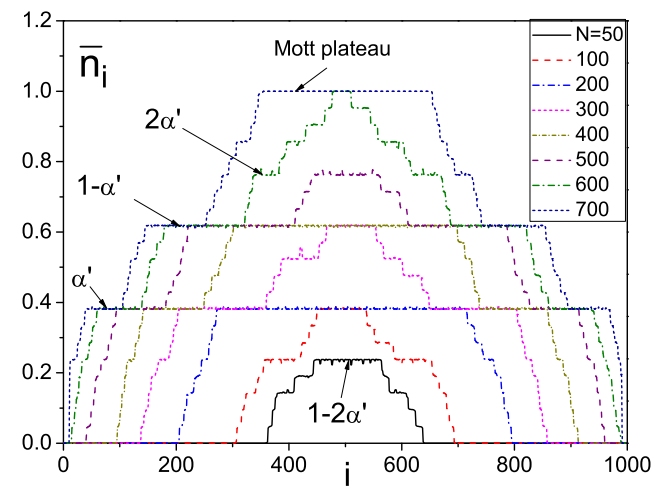

FIG. 2: (Color online) Local average density profiles for systems with different particle number (characteristic density). The other parameters of system are 1000 sites, $V_{I}=2.4$, $\alpha=(\sqrt{5}-1) / 2$ and $V_{H}=2.5 \times 10^{-5}$.

files for three systems with the same characteristic density relative to the scaled position $x=\left(i-i_{0}\right) / \zeta$. They are almost identical except of tiny differences caused by the setting of $M=10$ for all three systems with different length scale $\zeta$. So for systems with different particle number and strength of the harmonic trap, Anderson plateaus are the same so long as these systems have the same characteristic density. From now on, we fix the strength of harmonic trap and change particle numbers to achieve different characteristic densities. By doing this we don't need to scale the position for comparison among different systems. As shown in Fig,2, we plot local average density profiles for systems with fixed $V_{H}$ and different particle numbers (different $\widetilde{\rho}$ ). Here we choose $V_{I}>2$ to generate as many Anderson plateaus as possible in local average density profiles. Due to the incommensurate potential taking the form of $\cos (2 \pi \alpha i)$, for any $\alpha \in(-\infty, \infty)$ one can always choose a corresponding $\alpha^{\prime} \in[0,0.5)$ which generates the same incommensurate potential [13]. After counting on the locations of Anderson plateaus, we find that the height of Anderson plateaus is decided by $\alpha^{\prime}$ with values $\alpha^{\prime}, 1-\alpha^{\prime}, 2 \alpha^{\prime}, 1-2 \alpha^{\prime}, 2\left(1-\alpha^{\prime}\right), 1-2\left(1-\alpha^{\prime}\right), 4 \alpha^{\prime}, 1-4 \alpha^{\prime}, \ldots$, if the values are in the range of $(0,1)$. The Anderson plateaus with $\bar{n}_{i}=\alpha^{\prime}, 1-\alpha^{\prime}, 2 \alpha^{\prime}, 1-2 \alpha^{\prime}$ are the most important as they are wide enough and easy to appear for $V_{I}<2$. So heights of Anderson plateaus are totally decided by $\alpha^{\prime}$, while the width and the existence of the Anderson plateau are associated with $V_{I}$ and the characteristic density $(\widetilde{\rho})$. For system with high characteristic density, except of the existence of Anderson plateaus, there is a Mott plateau in the trap center characterized by $n_{i}=1$.

Finite-temperature density distribution and quantum criticality.- The finite-temperature density distribution 
can be calculated by

$$
n_{i}(T)=\frac{1}{Z} \sum_{n=1}^{N_{s}} e^{-E_{n} / k_{B} T}\left\langle\Psi_{\mathrm{HCB}}^{n}\left|\hat{b}_{i}^{\dagger} \hat{b}_{i}\right| \Psi_{\mathrm{HCB}}^{n}\right\rangle,
$$

where $N_{s}=L ! /(L-N) ! N !$ is the number of states, $E_{n}$ is the energy of eigenstate $\Psi_{\mathrm{HCB}}^{n}$, and $Z=\sum_{n=1}^{N_{s}} e^{-E_{n} / k_{B} T}$ is the canonical partition function. By using the exact numerical method in Ref. 19], we can calculate the finitetemperature properties of the hard-core bosons in incommensurate lattice very efficiently. In Fig 3, we display the local average density profiles for systems with different temperatures. The obvious plateau at zero temperature tends to vanish with the increase in the temperature. Consequently, the sharp boundary for superfluid and Anderson localized phases is destroyed by temperature fluctuations. If the temperature is low and located in the quantum critical regime, the density distributions for different temperatures should fulfil some universal scaling laws around the quantum phase transition point.

Before going to the analysis on the quantum criticality, we would like to introduce briefly the general theory for the quantum criticality. Given the equation of state $n=n(T, \mu)$ for a system with density $n$, temperature $T$ and chemical potential $\mu$, then near the zero-temperature phase transition point $\mu=\mu_{c}$, it was shown [8, 15] that when the dimensionality of the system is below a critical dimension, the following universal relation exists:

$$
n(\mu, T)-n_{r}(\mu, T)=T^{\frac{d}{z}+1-\frac{1}{\nu z} \Omega\left(\frac{\mu-\mu_{c}}{T^{1 / \nu z}}\right),}
$$

with $n_{r}(\mu, T)$ being the regular part of the density, $d$ the dimensionality of the system, $\nu$ the correlation length exponent, and $\mathrm{z}$ the dynamical exponent. $\Omega(x)$ is a universal function which describes the singular part of the density near criticality. If $n_{r}(\mu, T)$ in the above equation is known for one or more phases (such as vacuum and Mott insulator), Eq.(44) would be very useful for determining the quantum phase transition point. After scaling the density with the $T$ term, we get $A(\mu, T)=$ $T^{-\frac{d}{z}-1+\frac{1}{\nu z}}\left(n(\mu, T)-n_{r}(\mu, T)\right)$. If we plot $A(\mu, T)$ versus $\mu$ for a system at different temperatures, then curves with different temperatures will intersect at the same point $\mu=\mu_{c}$. With this scaling method, we can detect critical points of phase transitions at zero temperature from none-zero-temperature density profiles. Once $\mu_{c}$ is determined, we can scale the chemical potential $\widetilde{\mu}=\left(\mu-\mu_{c}\right) / T^{\frac{1}{\nu z}}$. By plotting $A(\mu, T)$ versus $\widetilde{\mu}$ for a system at different temperatures, all curves collapse into a single one (the universal function $\Omega(x)$ ). It was shown that $d=1, z=2$ and $\nu=1 / 2$ for both the vacuum-superfluid and superfluid-Mott phase transition of hard-core bosons [15].

For hard-core bosons in the $1 \mathrm{D}$ incommensurate lattice within a harmonic trap, there may exist vacuumsuperfluid, superfluid-BG, and even the superfluid-Mott

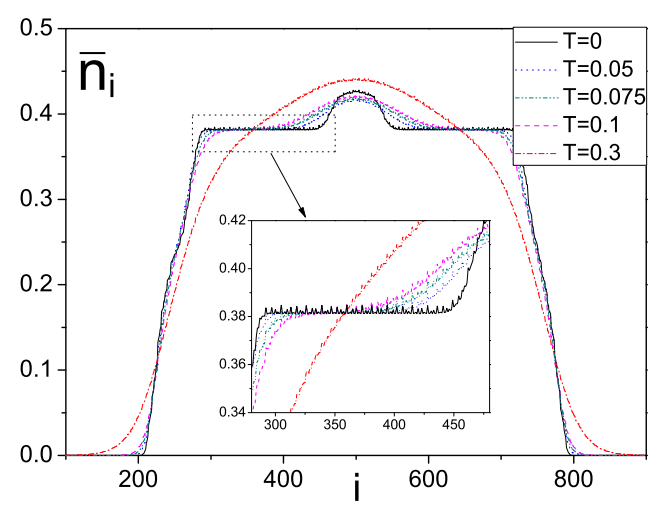

FIG. 3: (Color online) Local average density profiles for systems with different temperatures. Insert: enlargement of the Anderson-plateau area. The system is with 1000 sites, 200 bosons, $V_{I}=1.3, \alpha=(\sqrt{5}-1) / 2$ and $V_{H}=2.5 \times 10^{-5}$.

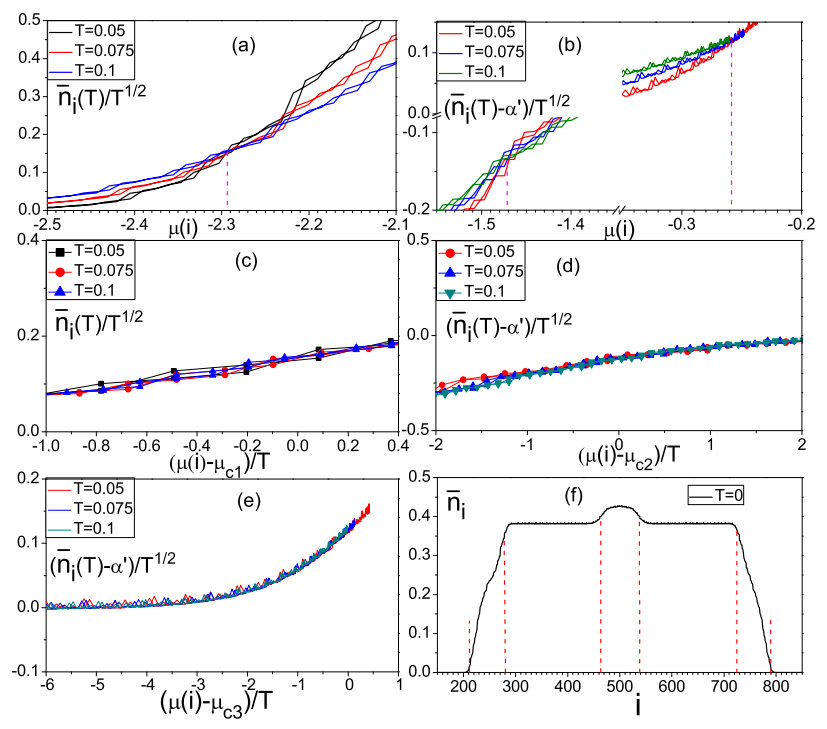

FIG. 4: (Color online) (a,b): Scaled local average density profiles vs $\mu(i)$ at different temperatures. (c,d,e): Scaled local average density profiles vs scaled chemical potential at different temperatures for $\mu_{c 1}(\mathrm{c}), \mu_{c 2}(\mathrm{~d}), \mu_{c 3}(\mathrm{e})$. (f): The local average density profile of the system at zero temperature together with the critical point $i_{c}$ got from the scaling method. The system is with 1000 sites, 200 bosons, $V_{I}=1.3$, $\alpha=(\sqrt{5}-1) / 2$ and $V_{H}=2.5 \times 10^{-5}$.

insulator phase transition as the position $i$ changing from sides to the center. If we replace the chemical potential $\mu$ in Eq.(44) by $\mu(i)=\mu-V_{H}(i)$, where $V_{H}(i)$ is the harmonic trap potential and $\mu$ is determined by $\sum_{i} n(\mu(i), T)=N$, then $\mu(i)$ will drive the transition from one phase to the other phase. Now we explore the quantum criticality of the disordered Bose system by using the local average density distribution. We find that there exist similar scaling laws described by Eq. (414) but with the density distribution in Eq.(4) being replaced by the local average density distribution. To give con- 


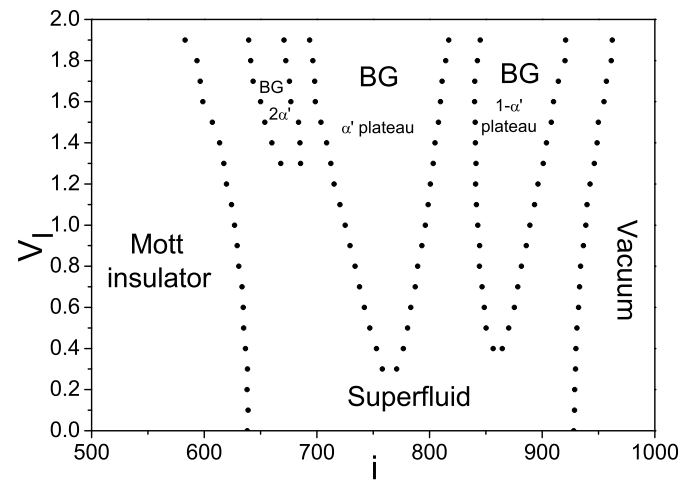

FIG. 5: The right half of the phase diagram at zero temperature in $V_{I}$-position plane of the system with 1000 sites, 600 bosons, $\alpha=(\sqrt{5}-1) / 2$ and $V_{H}=2.5 \times 10^{-5}$.

crete examples, we consider a system with 200 hard-core bosons, $V_{I}=1.3, \alpha=(\sqrt{5}-1) / 2$ and $V_{H}=2.5 \times 10^{-5}$, for which there is no Mott insulating plateau in the local average density profile as the characteristic den-

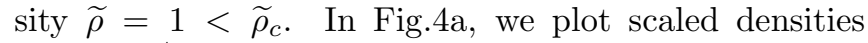
$\bar{n}_{i}(T) / T^{1 / 2}$ for different temperatures around the edge of the density profile, where $\bar{n}_{i}(T) \equiv \bar{n}\left(\mu_{i}, T\right)$. Different curves intersect at the point $\mu_{c 1}(i)=-2.293$, which is just the zero-temperature vacuum-superfluid transition point and occurs at sites $i_{c 1}=788.2$ and 212.8 via $\mu(i)=\mu-V_{H}(i)$.

From Fig 3 we know that Anderson plateaus appear at $\bar{n}_{i}=\alpha^{\prime}=0.38197$. By taking the regular part $\bar{n}_{r}=\alpha^{\prime}$, in Fig $4 \mathrm{~b}$ we plot scaled densities $\left(\bar{n}_{i}(T)-\alpha^{\prime}\right) / T^{1 / 2}$ versus $\mu(i)$ around the regime of Anderson plateau for different temperatures. It is clear that the different curves intersect at two points, which indicates that the superfluidBG phase transitions occur at $\mu_{c 2}(i)=-1.472$ and $\mu_{c 3}(i)=-0.258$ or equivalently at $i_{c 2}=724$ and 277 , and at $i_{c 3}=537.6$ and 463.4. To display the universal scaling functions, we plot scaled local average densities at different temperatures against scaled chemical potentials

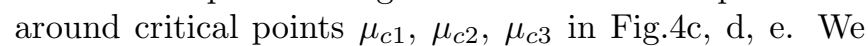
observe that curves for different temperatures indeed collapse onto a single curve after scaling, except that there are oscillations on the curves caused by the incommensurate lattice. In Fig [4 we show the zero-temperature local average density profile in contrast to critical points determined by the scaling method and marked by dash lines.

Through the analysis of quantum criticality, we can map out the zero temperature phase diagram from the nonzero-temperature local average density distributions. As an example, we show the phase diagram of the system with 1000 sites, 600 bosons, $\alpha=(\sqrt{5}-1) / 2$ and $V_{H}=2.5 \times 10^{-5}$ in the $V_{I}$-position plane, which has the characteristic density $\widetilde{\rho}=3>\tilde{\rho}_{c}$. As shown in Fig馬, without the incommensurate potential the system has a structure with the Mott insulator in the center surrounding by the superfluid phase on two sides. As $V_{I}$ increases, Anderson plateaus appear in the local average density profiles and the particles in them are in BG phase. With further increase in $V_{I}$, more plateaus appear and become wider. Furthermore, the size of Mott insulator decreases but the system size grows slowly as $V_{I}$ increases.

In summary, using the Bose-Fermi mapping, we study the universal properties and quantum criticality of onedimensional hard-core bosons on incommensurate optical lattices within harmonic traps. By calculating finitetemperature density distributions exactly, we unveil universal scaling relations of local average density distributions in the quantum critical regime. The zero temperature phase diagram is then determined from nonzero temperature local average density profiles with help of the analysis of quantum criticality.

We thank Xi-Wen Guan and Qi Zhou for helpful discussions. This work has been supported by NSF of China under Grants No.10821403 and No.10974234, 973 grant and National Program for Basic Research of MOST.

* Electronic address: schen@aphy.iphy.ac.cn

[1] B. Paredes, et. al., Nature 429, 277 (2004).

[2] T. Kinoshita, et. al., Science 305, 1125 (2004).

[3] E. Haller, et. al., Science 325, 1224 (2009).

[4] J. Billy et al., Nature (London) 453, 891 (2008).

[5] G. Roati et al., Nature (London) 453, 895 (2008).

[6] B. Deissler et al., Nat. Phys. 6, 354 (2010).

[7] M. Pasienski, D. McKay, M. White and B. DeMarco, Nat. Phys. 6, 677 (2010).

[8] M. P. A. Fisher, P. B. Weichman, G. Grinstein, D. S. Fisher, Phys. Rev. B 40, 546 (1989).

[9] T. Giamarchi and H. J. Schulz, Phys. Rev. B 37, 325 (1988).

[10] N. V. Prokofev and B. V. Svistunov, Phys. Rev. Lett. 80, 4355 (1998); S. Rapsch, U. Schollwöck, and W. Zwerger, Europhys. Lett. 46, 559 (1999).

[11] L. Fontanesi, M. Wouters, and V. Savona, Phys. Rev. Lett. 103, 030403 (2009); V. Guraie, G. Refael and J. T. Chalker, Phys. Rev. Lett. 101, 170407 (2008).

[12] A. De Martino, M. Thorwart, R. Egger, and R. Graham, Phys. Rev. Lett. 94, 060402 (2005).

[13] X. Cai, S. Chen, and Y. Wang, Phys. Rev. A 81, 023626 (2010).

[14] X. Cai, S. Chen, and Y. Wang, Phys. Rev. A 81, 053629 (2010).

[15] Q. Zhou and T. L. Hou, arXiv:1006.1174 (2010).

[16] K. R. A. Hazzard and E. J. Mueller, arXiv:1006.0969.

[17] X. W. Guan and T. L. Ho, arXiv: 1010.1301.

[18] M. Rigol and A. Muramatsu, Phys. Rev. A 72, 013604 (2005).

[19] M. Rigol, Phys. Rev. A 72, 063607 (2005).

[20] M. Rigol and A. Muramatsu, Phys. Rev. A 70, 043627 (2004). 